\title{
Investigation of Influence of Mixed Configurations on Performance of WDM-PON
}

\author{
Inna Kurbatska ${ }^{1}$, Anita Alsevska ${ }^{1}$, Lilita Gegere ${ }^{1}$, Vjaceslavs Bobrovs ${ }^{1}$ \\ ${ }^{1}$ Institute of Telecommunications, Riga Technical University \\ Azenes 12, LV-1048, Riga, Latvia \\ inna.kurbatska@rtu.lv
}

\begin{abstract}
This paper aims at assessing of implementation of the concept of mixed systems in wavelength-divisionmultiplexing passive optical networks (WDM-PONs). At the first stage, the authors evaluate performance of two equalchannel transmission systems with non-return-to-zero (NRZ) and duobinary (DB) coding in terms of Q-factor, minimal channel spacing and supported data rate. At the second stage, two configurations of mixed transmission systems are proposed. The system with unequal channel spacing and multiple modulation formats (MMFs) as well as the system with mixed line rates (MLRs) and MMFs are investigated. The obtained results reveal that the impact of implementation of both systems is positive and concept of mixed transmission system can be considered as a potential solution for the increase of spectral efficiency (SE) of WDM-PON transmission system.
\end{abstract}

Index Terms-Duobinary; channel spacing; intensity modulation; WDM networks.

\section{INTRODUCTION}

Nowadays, the expansion and variety of new broadband services along with their rapid advance increase the demands of users for internet traffic. Consequently, internet service providers (ISPs) need to introduce new technologies for increasing the capacity of existing transmission system [1]. Furthermore, the advancing of access network plays an important role in providing internet traffic available for consumers.

Considering that the wavelength-division-multiplexed passive optical network (WDM-PON) is one of the most likely candidate for applying next-generation PON2 (NGPON2), we focus on the increasing of transmission throughput of WDM-PON in present research [2].

The one of the most intensively studied solutions of rising the total transmission capacity of WDM system is the increase of its spectral efficiency (SE) [1], [3].

There are two popular solutions for increasing SE of existing WDM transmission systems. Using higher data rates and applying narrower channel spacing, both allow transmitting a greater number of bits using one hertz of available bandwidth [1], [4]. These concepts are considered as the most cost-effective because of the ability to maintain maximum of already deployed infrastructure [3]. However, several technological issues tend to appear with the introducing of above-mentioned approaches. Firstly, increasing of the data rate requires new and more expensive

Manuscript received 15 September, 2016; accepted 17 December, 2017. equipment as well as increasing power consumption of WDM transmission system [5], [6]. It is important to take into account that traditionally used intensity modulation with non-return-to-zero (NRZ) coding is not able to provide sufficient quality of the signal with the increasing of data rate or reducing channel spacing. It may also result in a shorter reach due to increasing of the optical transmission impairments [4]-[6]. Consequently, in some cases we have to consider modulation techniques with better SE and reachrate product. For example, although $10 \mathrm{Gbit} / \mathrm{s}$ systems typically utilize NRZ intensity modulation, it is necessary to employ more advanced modulation formats, such as duobinary (DB) or phase shift keying (PSK) modulation to move to the rate of $40 \mathrm{Gbit} / \mathrm{s}$ and higher [5]. Nevertheless, the applying of new modulation formats requires a change of optical transmitters and, for some modulation formats also a change of receivers. Consequently, it causes the growth of expenses for network enhancement.

The applying of the concept of mixed transmission system is an effective solution for this trade-off between SE and expenses on the development of the transmission system. In the case of mixed transmission system, new data rates or modulation formats can be applied only for the part of channels ensuring lower expenses. Therefore, the implementation of mixed transmission system allows providing maximal SE keeping necessary signal quality with limited expenses. This is assured changing a percentage of channels with high data rate, another modulation formats or minimal channel spacings. Consequently, mixed WDM system can be considered as an intermediate for gradual transition to higher data rate transmission system as well as for transition to another modulation format. The necessity for transmission of channels with multiple modulation format (MMFs) or mixed-line rates (MLRs) may arise also as a result of different strategies of transmission network's infrastructure development performed by different ISPs [3], [4].

The aim of our research is to evaluate coexistence of MMFs and MLRs applied in the case of transitional stage to higher data rates with fewer expenses or in the case of network enhancement by one of the multiple operators. We investigate how the applying of MMFs, MLRs and unequal channel spacings influences the performance of WDM-PON transmission system, comparing the performance of the systems with mixed configurations with the performance of WDM-PON transmission system with equal channels. We 
also evaluate the SE of mixed WDM-PON systems in comparison with WDM-PONs with the same modulation formats, data rates and channel spacings for all channels.

As it is an initial-stage research, we are evaluating separately the performance of MLR system and the system with unequal channel spacings in both cases applying MMFs. That allows investigation of influence of applying of each concept.

We utilize the Duobinary (DB) intensity modulation as the second modulation format, which we use for applying minimal channel spacing in the mixed transmission system with unequal channel spacings as well as for applying $40 \mathrm{Gbit} / \mathrm{s}$ data rate in the MLR WDM-PON. We introduce $\mathrm{DB}$ modulation format into the traditional NRZ system implementing the concept of mixed transmission system with MMFs.

Our research is realized in two stages. In the first stage, we are investigating the performance of the base transmission system, which we consider as an already existing. This is the 16 channel $10 \mathrm{Gbit} / \mathrm{s}$ WDM-PON transmission system with NRZ intensity modulation. We also evaluate the performance of $10 \mathrm{Gbit} / \mathrm{s}$ DB transmission system, investigating the benefits of DB WDM-PON in comparison with NRZ WDM-PON. We determine the minimum allowable and equal frequency intervals for both systems as well as investigate the performance of the systems at higher data rate $(40 \mathrm{Gbit} / \mathrm{s})$. This is performed in order to substantiate expedience of implementing of DB in our investigated WDM-PON as well as investigate the capabilities of both formats for their implementation in mixed transmission systems.

In the second stage, we change NRZ coding to DB for the half of channels. At the beginning, we apply the mixed transmission system with unequal channel spacing and MMFs. After that, we investigate the mixed transmission system with MLRs and MMFs. We evaluate how the performance of the system changed in comparison with the NRZ system in the case of system with MLR with MMF and in the case of the system with unequal channel spacings. Based on obtained results we investigate the impact of applying the mixed systems comparing the performance with the systems investigated in the first stage. We also evaluate the opportunities given by this transitional solution.

\section{STATE OF ART}

Quite recently, considerable attention has been paid to different concepts of mixed systems.

MLR systems, systems with non-equal channel spacings as well as systems with MMFs have been investigated. Some studies have focused also on combination of abovementioned solutions.

The most of researches demonstrated that mixed transmission systems can be successfully applied without negative impact on the performance of WDM transmission system. Moreover, the utilizing of mixed configurations can increase the SE, cost-efficiency and also energy-efficiency of transmission system.

For example, according to the analysis of logical network layer, that have been performed in [6], MLR networks with MMFs are more cost effective solutions in comparison with equal-channel networks. In [4] authors propose several power efficient and cost-efficient solutions for implementation of MLR concept in high-speed long haul networks. Authors in [7] reveal that the applying unequally channel spacing in proper configuration provides increasing the SE of WDM backbone network with MLRs and MMFs. Authors in [8] also demonstrate the successful implementation of MLR transmission system with unequal channel spacing, comparing the performance of NRZ. DB and NRZ differential phase shift keying (NRZ-DPSK) modulation formats in $40 \mathrm{Gbit} / \mathrm{s}$ transmission systems.

However, the influence of applying of mixed transmission systems can be both positive and negative. For example, the results obtained in [5] suggest that applying of MLR and MMF systems can significantly influence the performance of WDM transmission system. Authors indicate that simultaneously transmitted optical signals with different modulation formats can lead to considerable degradations in signal quality and, consequently, to reductions in the transmission reach up to $25 \%$ [5]. For this reason it is crucial to evaluate whether the concept of mixed transmission system can be applied without decreasing the performance in comparison this the existing transmission system.

However, all above-mentioned researches are devoted to long-haul networks based on active optical networks (AON) with dispersion compensation. To the authors' best knowledge, there are no publications available in the literature that discuss the issue of applying mixed transmission systems in access networks. Whereas the above-mentioned reasons for applying of mixed systems as gradual transition to higher data rates and shared use of infrastructure by different ISPs are crucial also for this network segment. Moreover, such benefits of mixed solutions as SE, cost efficiency and energy efficiency are important also for access networks. However, access networks are based on WDM-PON, which do not assume the utilizing of optical amplifiers. It is also more cost-efficient not to use dispersion compensators for such small transmission distances (up to $20 \mathrm{~km}$ ). Due to these significant differences in the structures of transmission systems for long-haul networks and access networks, it is not correct to assume that the impact of introducing the mixed configurations in WDM-PON access networks will be the same as the impact of introducing it in long-haul WDM networks that have already been investigated. That is why it is important to evaluate whether the concepts of mixed configurations demonstrated in above-mentioned researches for long-haul WDM transmission systems, can be as successfully implemented in WDM-PON transmission systems for access networks. So that our research focuses on applying of mixed transmission systems in WDM-PONs.

The choice of DB coding for implementation of our mixed solutions is also based on researches that have been performed previously. Large number of studies demonstrate that $\mathrm{DB}$ modulation format is a promising solution for providing higher data rates and smaller channel spacing, because of its high chromatic dispersion tolerance and narrow spectral width. For example authors in [9] reveal that DB demonstrated its superiority over the other investigated 
intensity formats (NRZ, Return to Zero, Carrier-Suppressed Return to Zero) achieving minimal channel spacing equal to $12.5 \mathrm{GHz}$ at $10 \mathrm{Gbit} / \mathrm{s}$ WDM system. DB was also more efficient than other formats at $40 \mathrm{Gbit} / \mathrm{s}$ transmission rate. Furthermore, according to the results demonstrated in [8] DB was the most successful format for the utilization in MLR systems in comparison with NRZ and DPSK. In addition authors in [10] report, that DB demonstrated higher SE in comparison with NRZ, RZ and CSRZ in the long-haul high-data rate system with unequal channel spacing and MMFs. Moreover, the transmitter of DB is much less complicated in comparison with advanced modulation formats such as PAM, QAM and QPSK. As it is reported in [11], DB modulation format offers the best receiver sensitivity and is more power efficient in comparison with PAM-4 in 40 Gbit/s WDM-PON transmission systems. It is also important that $\mathrm{DB}$ do not require the change of receiver, because it is possible to use the same receiver as in the case of NRZ coding.

\section{Simulation Methods AND Simulation SETUP}

The results were obtained using OptSim simulation software. The calculations in OptSim are performed using time domain split step (TDSS) method, which is based on dividing fiber into small spans for separate calculating of linear and nonlinear effects with small error probability. The method is described in details in [12].

Figure 1 illustrates the structure of our simulation system, which corresponds to the typical WDM-PON structure for downstream transmission.

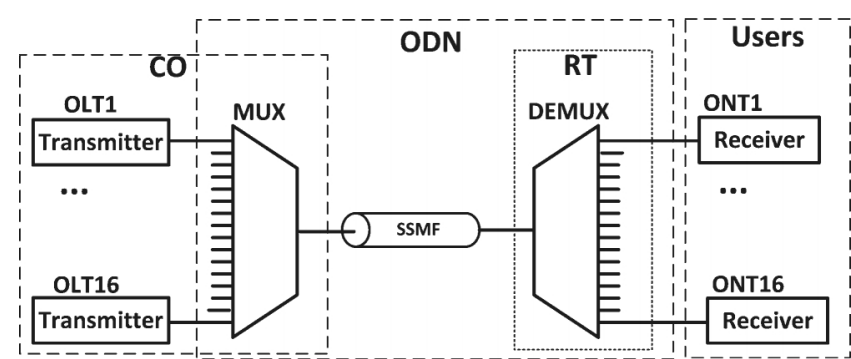

Fig. 1. The structure of the investigated 16 channel WDM-PON transmission system.

Optical line terminal (OLT) consists of optical transmitter. The structure of transmitter depends on modulation format. Nonetheless, both transmitters are based on continuous wave (CW) laser with $+6 \mathrm{dBm}$ output power and $0.1 \mathrm{MHz}$ linewidth [13]. Optical network terminal (ONT) comprises of the optical receiver based on PIN photodiode with -18.5 $\mathrm{dBm}$ sensitivity [14]. The optical distribution network (ODN) includes optical fibre (G.652.D), WDM multiplexer and demultiplexer. In the case of the systems with the equal channels, arrayed waveguide grating (AWG) multiplexer and demultiplexer is used. In the case of mixed system, it is necessary to use optical filters, as well as splitters and combiners to assure different channel spacing and appropriate bandwidth for each modulation format. As our simulation results reveal, the change of AWG multiplexer to the combination of optical filter and optical combiner as well as the change of AWG multiplexer to the combination of optical filter and optical splitter do not significantly influence performance of the system.

\section{RESULTS AND DISCUSSION}

At the beginning of the first stage, we determined the minimal channel spacings realizable by the system with NRZ coding and by the system with DB coding. The performance of the both systems was evaluated in the terms of Q-factor. The value of $\mathrm{Q}$-factor equal to $16.95 \mathrm{~dB}$, corresponding to BER equal to $10^{-12}$ was chosen as a preliminary threshold [3]. Nevertheless, as the satisfactory value of Q-factor depends on the requirements to the reliability of the system, it should be taken into account, that in some applications lower value of Q-factor might be allowed [4].

We started by investigating transmission at $20 \mathrm{~km}$ long distance applying fixed channel intervals specified in ITU-T G.694.1. For $100 \mathrm{GHz}, 50 \mathrm{GHz}$ and $25 \mathrm{GHz}$ channel spacings both NRZ and DB, demonstrated satisfactory performance (Q-factor higher than $16.95 \mathrm{~dB}$ ). However, for $12.5 \mathrm{GHz}$ channel spacing NRZ format demonstrated too low value of the Q-factor (Q-factor equal to $6.02 \mathrm{~dB}$ ). Results demonstrated by DB are significantly better (Qfactor of the worst channel is equal to $14.56 \mathrm{~dB}$ ). That corresponds to the results obtained in [9], where with the utilizing of fixed ITU-T G.694. frequency grid, the minimal achievable channel spacing for NRZ was $25 \mathrm{GHz}$, while for DB it was $12.5 \mathrm{GHz}$.

As one can see at Fig. 2 results demonstrated by different channels of DB transmission system are different (Q-factor is in the range from $14.56 \mathrm{~dB}$ to $16.91 \mathrm{~dB}$ ). These results allow considering the applying of DB with channel spacing equal to $12.5 \mathrm{GHz}$ in the system with unequal channel spacings may improve the performance, because not all of the channels will have such small spacing.

To achieve the maximal possible SE, we determined the minimal channel spacing applying that both system will work with sufficiently high Q-factor, using flexible WDM frequency grid described in ITU-T G.694.1. We decreased the $25 \mathrm{GHz}$ spacing by $6.5 \mathrm{GHz}$. As a result, we investigated that the minimal channel spacing achieved by NRZ system is $18.75 \mathrm{GHz}$.

Figure 2 presents the Q-factor values for all channels of NRZ and DB systems.

All values Q-factor values higher than 18.3 (corresponding to $\mathrm{BER}=10^{-16}$ ) are rounded to this value. As one can see, the performance of $\mathrm{DB}$ modulation format is better, but for both formats Q-factor is higher than $16.95 \mathrm{~dB}$, so we can assume that for $18.75 \mathrm{GHz}$ channel spacing the performance of NRZ modulation format is satisfactory.

The SE calculated for NRZ transmission system with $18.75 \mathrm{GHz}$ channel spacing is $0.61 \mathrm{bit} / \mathrm{s} / \mathrm{Hz}$, but for $\mathrm{DB}$ system with $12.5 \mathrm{GHz}$ channel spacing is equal to $0.85 \mathrm{bit} / \mathrm{s} / \mathrm{Hz}$.

At the second part of the first stage, we evaluated how the increase of the transmission rate to $40 \mathrm{Gbit} / \mathrm{s}$ influences the performance of investigated transmission systems. We applied constant $100 \mathrm{GHz}$ channel spacing for $40 \mathrm{Gbit} / \mathrm{s}$ transmission system in current research. Both NRZ and DB transmission systems demonstrated low Q-factor (equal to $6.02 \mathrm{~dB}$ ) for $20 \mathrm{~km}$ transmission distance. 


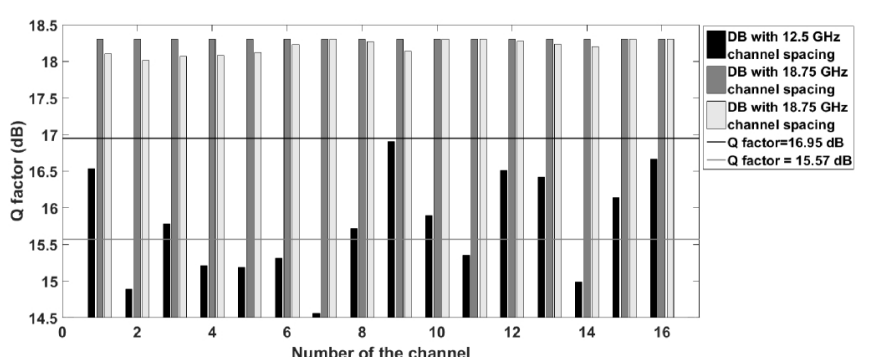

Fig. 2. Q-factor performance of the channels of investigated DB transmission system with $12.5 \mathrm{GHz}$ channel spacing and of DB and NRZ transmissions systems with $18.75 \mathrm{GHz}$ channel spacing at $20 \mathrm{~km}$ reach

However, decreasing the distance, the difference in the performance of both systems is significant. System with DB coding demonstrated ability to provide transmission at 12 $\mathrm{km}$ long distance with the Q-factor higher than $16.95 \mathrm{~dB}$. Whereas NRZ modulation format demonstrated the low values of $\mathrm{Q}$-factor also with the reach decreasing. These results are consistent with the theoretical characteristics of NRZ and DB modulation formats: DB has better dispersion tolerance in comparison with NRZ, which results in ability to provide transmission with $40 \mathrm{Gbit} / \mathrm{s}$ data rate without using dispersion compensation. However, the maximal reach is limited even for DB transmission systems.

Table I summarizes the results of the first stage, representing the Q-factor values for the worst channels.

TABLE I. THE PERFORMANCE OF THE EQUAL-CHANNEL NRZ

\begin{tabular}{|c|c|c|c|c|}
\hline \multicolumn{5}{|c|}{ AND DB TRANSMISSION SYSTEMS. } \\
\hline Coding & $\begin{array}{c}\text { Data rate } \\
\text { (Gbit/s) }\end{array}$ & $\begin{array}{c}\text { Channel } \\
\text { spacing (GHz) }\end{array}$ & $\begin{array}{c}\text { Transmission } \\
\text { distance (km) }\end{array}$ & $\begin{array}{c}\text { Q- } \\
\text { factor } \\
(\mathbf{d B})\end{array}$ \\
\hline NRZ & 10 & 12.5 & 20 & 6.02 \\
\hline DB & 10 & 12.5 & 20 & 14.56 \\
\hline NRZ & 10 & 18.75 & 20 & 18.01 \\
\hline DB & 10 & 18.75 & 20 & 18.38 \\
\hline NRZ & 40 & 100 & 20 & 6.02 \\
\hline DB & 40 & 100 & 20 & 6.02 \\
\hline NRZ & 40 & 100 & 12 & 6.02 \\
\hline DB & 40 & 100 & 12 & 17.64 \\
\hline
\end{tabular}

Based on the first stage, we proposed two mixed solutions for WDM-PON.

First solution is MMF system with unequal channel intervals. Our proposed configuration of MMFs and channel spacings is illustrated at Fig. 3 using the output spectrum of the system.

The configuration, where two DB channels follow two NRZ channels, allowed providing $12.5 \mathrm{GHz}$ channel spacing between two adjacent DB channels, keeping the $18.5 \mathrm{GHz}$ spacing between NRZ and NRZ-DB pairs. As we investigated by simulations, it is impossible to provide the smaller channel spacing between NRZ pair or between NRZ and DB, without significant decrease of quality of NRZ channel. The SE of this system is equal to $0.62 \mathrm{bit} / \mathrm{s} / \mathrm{Hz}$ but Q-factor values of channels can be found in Fig. 4.

As one can see, the results have improved for both DB and NRZ. In the mixed system, Q-factor of the worst DB channel is $15.9 \mathrm{~dB}$ in comparison with Q-factor of the worst channel equal to $14.56 \mathrm{~dB}$ in $\mathrm{DB}$ system with $12.5 \mathrm{GHz}$ channel spacing. The most likely explanation of the increasing of Q-factor is the applying of mixed channel spacings, implying the $12.5 \mathrm{GHz}$ only with the one of two side channels. Q-factor of NRZ channels in mixed system is not lower than $18.28 \mathrm{~dB}$ in comparison with Q-factor of $18.01 \mathrm{~dB}$ demonstrated by NRZ $18.75 \mathrm{GHz}$ system. This can be explained, by employment of narrow-spectrum DB modulation format for one of two side channels.

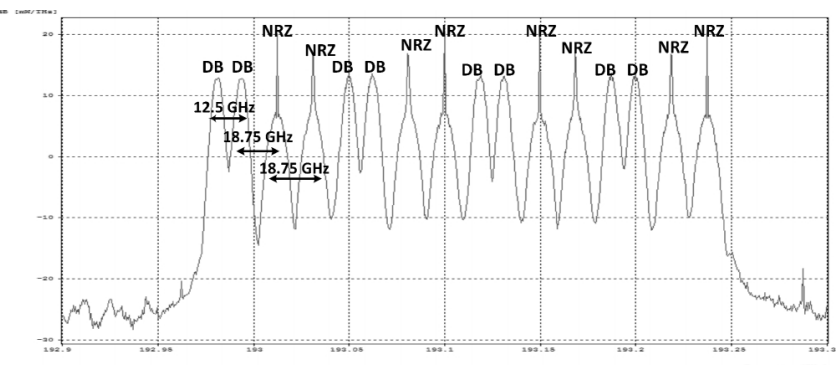

Fig. 3. The output spectrum of the mixed system with MMF and unequal channel spacings.

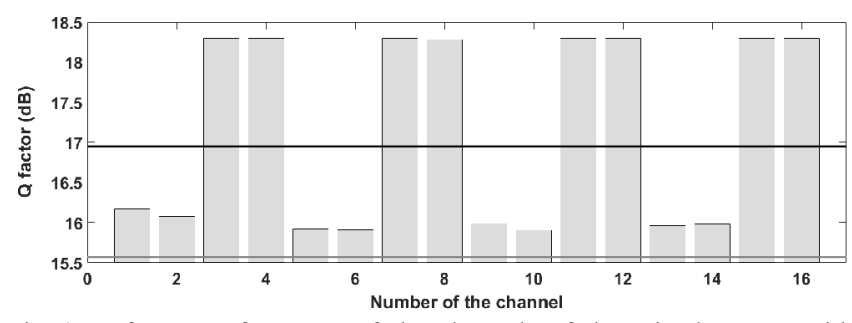

Fig. 4. Q-factor performance of the channels of the mixed system with MMF and unequal channel spacings at $20 \mathrm{~km}$ reach.

Simulating the scenario of partial applying of $40 \mathrm{Gbit} / \mathrm{s}$ channels in NRZ $10 \mathrm{Gbit} / \mathrm{s}$, our second proposed mixed WDM-PON system is demonstrated in the Fig. 5.

In this system one $40 \mathrm{Gbit} / \mathrm{s}$ DB channel follows one NRZ $10 \mathrm{Gbit} / \mathrm{s}$ channel. Obtained results are summarized at Fig. 6.

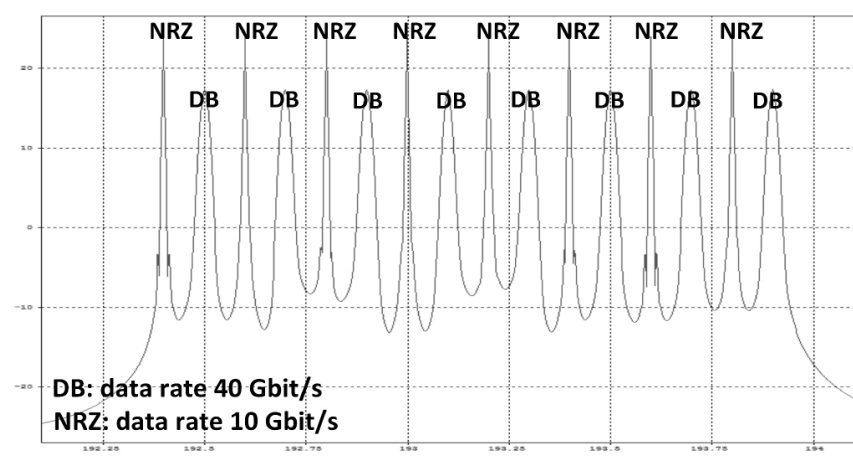

Fig. 5. The output spectrum of the mixed system with MMFs and MLRs.

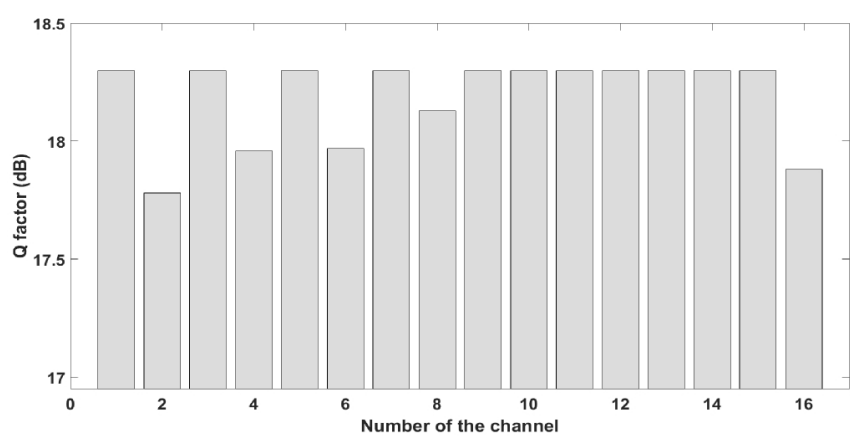

Fig. 6. Q-factor performance of the channels of the mixed system with MMF and MLRs at $12 \mathrm{~km}$ reach. 
As one can see, the minimal value of Q-factor among DB channels is $17.78 \mathrm{~dB}$ in comparison with the $17.64 \mathrm{~dB}$ demonstrated in system with all $40 \mathrm{Gbit} / \mathrm{s}$ DB channels. That means that the performance of DB have become better in the mixed line rate systems.

Performance of NRZ channels is very good, the presence of DB channels have not affected them. However, the SE of the system is only $0.27 \mathrm{bit} / \mathrm{s} / \mathrm{Hz}$ due to relatively big 100 $\mathrm{GHz}$ channel spacing. According to the results obtained in [8] for the long-haul MLR WDM system, it is possible to achieve higher $\mathrm{SE}(0.68 \mathrm{bit} / \mathrm{s} / \mathrm{Hz})$ by applying the unequal channel spacing for MLR system. Nonetheless, the SE demonstrated by equal-channel NRZ transmission system is even lower (equal to $0.1 \mathrm{bit} / \mathrm{s} / \mathrm{Hz}$ ) than demonstrated by our proposed MLR system, which means that introducing of high-data rate channels can significantly improve the spectral efficiency of the system. Moreover, very high values of Q-factor of the channels mean that there is a possibility for the decreasing the channel spacing.

\section{CONCLUSIONS}

The aim of our research was to investigate, how the applying of mixed configuration influences the performance of the WDM-PON. Two concepts of mixed transmission systems - system with MMFs and unequal channel spacing and MLR system with different MMFs- have been evaluated.

The obtained results have indicated that Q-factor demonstrated by the worst NRZ channel in the mixed transmission system with unequal channel spacings (equal to $18.3 \mathrm{~dB}$ ) is higher than the Q-factor of the worst channel in the $10 \mathrm{Gbit} / \mathrm{s} \mathrm{NRZ}$ transmissions system with $18.75 \mathrm{GHz}$ channel spacing $(18.01 \mathrm{~dB})$. The Q-factor of the worst NRZ channel obtained in the MLR transmission system $(18.28 \mathrm{~dB})$ is also higher than $18.01 \mathrm{~dB}$ Q-factor demonstrated with the reference system. That allows concluding, that the applying of both above mentioned concepts do not affect the performance of WDM-PON harmfully and even increase it.

The obtained results have also shown that the partial applying of channels with $40 \mathrm{Gbit} / \mathrm{s}$ data rate in WDM-PON increases the Q-factor of these channels in comparison with the system with the $40 \mathrm{Gbit} / \mathrm{s}$ data rate for all channels. The Q-factor of the worst $40 \mathrm{Gbit} / \mathrm{s}$ DB channel in MLR system is equal to $17.78 \mathrm{~dB}$ in comparison with $17.64 \mathrm{~dB}$ demonstrated by the system with all $40 \mathrm{Gbit} / \mathrm{s}$ DB channels. Based on these results, it is possible to conclude, that the applying of $40 \mathrm{Gbit} / \mathrm{s}$ data only for the part of the channels make easier meeting the demands on the performance of WDM-PON.

Our performed evaluation of the influence of applying of mixed configurations on the SE of WDM-PON revealed that both system with unequal channel spacing and the MLR system demonstrate the increase of SE in comparison with the systems with equal channels. The SE of our proposed $10 \mathrm{Gbit} / \mathrm{s}$ system with unequal channel spacing is $0.62 \mathrm{bit} / \mathrm{s} / \mathrm{Hz}$, that is by $83,87 \%$ higher than the SE of typical $100 \mathrm{GHz}, 10 \mathrm{Gbit} / \mathrm{s}$ WDM-PON (equal to $0.1 \mathrm{bit} / \mathrm{s} / \mathrm{Hz}$ ). However, applying of fixed, but minimum achievable channel spacing equal to $18.75 \mathrm{GHz}$ allows achieving only slightly worse SE than mixed configuration (equal to $0.61 \mathrm{bit} / \mathrm{s} / \mathrm{Hz}$ ). That means that the increase of SE mostly is ensured by optimizing channel spacing. The SE of our investigated MLR system is equal to $0.27 \mathrm{bit} / \mathrm{s} / \mathrm{Hz}$ that is $62 \%$ increase in comparison with $10 \mathrm{Gbit} / \mathrm{s}$ WDM-PON $(0.1 \mathrm{bit} / \mathrm{s} / \mathrm{Hz})$. Moreover, the sufficiently high value of Qfactor of the MLR system (equal to $17.78 \mathrm{~dB}$ ), allows to conclude that the SE of the system can be increased by the decreasing channel spacing still meeting minimal Q-factor requirements.

Summing up, this paper has demonstrated that the concepts of MLR, MMFs and unequal channel spacings can be successfully applied in WDM-PONs. However, further research on the simultaneous applying of these concepts is desirable for attainment of higher SE of WDM-PON.

\section{REFERENCES}

[1] A. Udalcovs, V. Bobrovs, I. Trifonovs, T. Celmins, "Investigation of maximum distance reach for spectrally efficient WDM system with mixed data rates and signal formats", Elektronika ir Electrotechnika, vol. 19, no. 1, pp. 87-92, 2013. [Online]. Available: http://dx.doi.org/ 10.1109/BCFIC.2012.6217979

[2] Next-Generation PON Evolution, Huawei Technologies Co., Ltd, pp. 24, 2010.

[3] A. Udalcovs, V. Bobrovs, "Investigation of spectrally efficient transmission for differently modulated optical signals in mixed data rates WDM systems", in Proc. IEEE Swedish Communication Technologies Workshop (Swe-CTW), 2011, pp. 7-12. [Online] Available: http://dx.doi.org/10.1109/Swe-CTW.2011.6082493

[4] A. Udalcovs, P. Monti, V. Bobrovs, R. Schatz, L. Wosinska, G. Ivanovs, "Spectral and energy efficiency considerations in mixedline rate WDM networks with signal quality guarantee", in Proc. 15th Int. Conf. Transparent Optical Networks (ICTON), 2013, pp. 1-7. [Online]. Available: http://dx.doi.org/10.1109/ICTON.2013.6602845

[5] K. Christodoulopoulos, K. Manousakis, E. Varvarigos, "Planning mixed-line-rate WDM transport networks", in Proc. 13th Int. Conf. Transparent Optical Networks, 2011, pp. 1-4. [Online]. Available: http://dx.doi.org/10.1109/ICTON.2011.5970835

[6] A. Nag, M. Tornatore, B. Mukherjee, "Optical network design with mixed line rates and multiple modulation formats", Journal of Lightwave Technology, vol. 28, no. 4, pp. 466-475, 2010. [Online]. Available: http://dx.doi.org/10.1109/JLT.2009.2034396

[7] A. Udalcovs, V. Bobrovs, "Investigation of spectrally efficient transmission for unequally channel spaced WDM systems with mixed data rates and signal formats", in Proc. 8th Int. Symposium on Communication Systems, Networks \& Digital Signal Processing (CSNDSP), 2012, pp. 1-4. [Online]. Available: http://dx.doi.org/ 10.1109/CSNDSP.2012.6292662

[8] R. Bajpai, S. Sengar, S. P. Singh, "Comparison of performance of a mixed line rate optical WDM network using different modulations at 40Gbps", Int. Conf. Advances in Computing, Communication, \& Automation (ICACCA), Spring, 2016, pp. 1-3. [Online]. Available: http://dx.doi.org/10.1109/ICACCA.2016.7578869

[9] R. Agalliu, M. Lucki, "System performance and limits of optical modulation formats in dense wavelength", Elektronika ir Electrotechnika, vol. 22, no. 2, pp. 123-129, 2016. [Online]. Available: http://dx.doi.org/10.5755/j01.eie.22.2.9599

[10] N. Sharma, R. Vij, N. Badhan, "Enhanced spectral efficiency for intensity modulated DWDM systems", 2015 Twenty First National Conf. Communications (NCC), Mumbai, 2015, pp. 1-6. [Online]. Available: http://dx.doi.org/10.1109/NCC.2015.7084818

[11] J. L. Wei, K. Grobe, C. Sanchez, E. Giacoumidis, H. Griesser, "Comparison of cost - and energy-efficient signal modulations for next generation passive optical networks", Optics Express, vol. 23, no. 22, pp. 28271-28281, 2015. [Online]. Available: http://dx.doi.org/10.1364/OE.23.028271

[12] OptSim User Guide, RSoft Design Group, Inc., pp. 459, 2010.

[13] Datasheet Co Brite DX1 laser, ID Photonics.

[14] Datasheet 10G receiver module, Amonics LTD. 